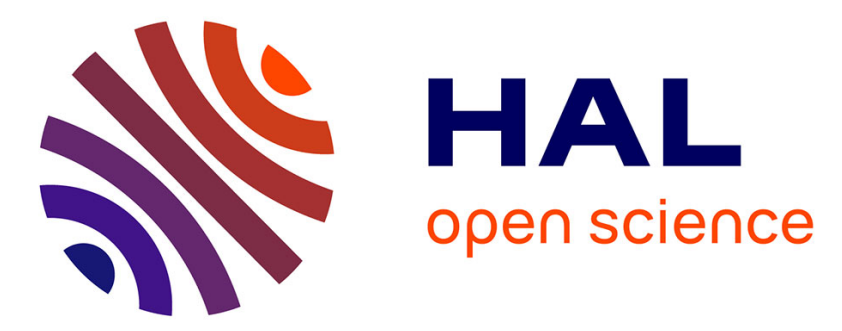

\title{
A Matsumoto-Yor property for Kummer and Wishart random matrices
}

Angelo Efoévi Koudou

\section{To cite this version:}

Angelo Efoévi Koudou. A Matsumoto-Yor property for Kummer and Wishart random matrices. Statistics and Probability Letters, 2012, 82 (11), pp.1903-1907. 10.1016/j.spl.2012.06.024 . hal01284058

\section{HAL Id: hal-01284058 \\ https://hal.science/hal-01284058}

Submitted on 7 Mar 2016

HAL is a multi-disciplinary open access archive for the deposit and dissemination of scientific research documents, whether they are published or not. The documents may come from teaching and research institutions in France or abroad, or from public or private research centers.
L'archive ouverte pluridisciplinaire HAL, est destinée au dépôt et à la diffusion de documents scientifiques de niveau recherche, publiés ou non, émanant des établissements d'enseignement et de recherche français ou étrangers, des laboratoires publics ou privés. 


\title{
A Matsumoto-Yor property for Kummer and Wishart random matrices
}

\author{
A. E. Koudou \\ Institut Elie Cartan, Laboratoire de Mathématiques, B.P. 239, \\ F-54506 Vandoeuvre-lès-Nancy cedex, \\ Efoevi.Koudou@iecn.u-nancy.fr
}

September 23, 2011

\begin{abstract}
For a positive integer $r$, let $I$ denote the $r \times r$ unit matrix. Let $X$ and $Y$ be two independent $r \times r$ real symmetric and positive definite random matrices. Assume that $X$ follows a Kummer distribution while $Y$ follows a non-degenerate Wishart distribution, with suitable parameters. This note points out the following observation: the random matrices $U:=\left[I+(X+Y)^{-1}\right]^{1 / 2}\left[I+X^{-1}\right]^{-1}[I+(X+$ $\left.Y)^{-1}\right]^{1 / 2}$ and $V:=X+Y$ are independent and $U$ follows a matrix beta distribution while $V$ follows a Kummer distribution. This generalizes to the matrix case an independence property established in Koudou and Vallois (2010) for $r=1$.
\end{abstract}

Keywords: Wishart distribution; Matsumoto-Yor property; Matrix Kummer distribution; Matrix Beta distribution.

\section{Introduction}

If $X, Y$ are two real positive and independent random variables and if $f:] 0, \infty[\rightarrow] 0, \infty[$ is a decreasing and bijective function, define $U:=f(X+Y)$ and $V:=f(X)-f(X+Y)$. For $f(x)=1 / x$, if $X$ follows a generalized inversed Gaussian (GIG) distribution and if $Y$ has a gamma distribution with a suitable matching of the parameters, then $U$ and $V$ are independent. This fact, partially observed in Matsumoto and Yor (2001) and completely proved in Letac and Wesołowski (2000), is known in the recent literature as the Matsumoto-Yor property. Furthermore, it characterizes the product of GIG and gamma laws, as shown in Letac and Wesołowski (2000).

In Koudou and Vallois (2010), other independence properties of the Matsumoto-Yor type have been proved, by observing that there are essentially four possible functions $f$, and characterizations of the corresponding laws have been given under the assumptions of existence and smoothness of densities (see also Koudou and Vallois, 2011, where these assumptions have been slightly relaxed). For $f(x)=\log (1+x)-\log x$ the corresponding 
independence property states the following: if $X, Y$ are independent random variables such that the law of is a Kummer law

$$
K^{(2)}(a, b, c)(d x):=C x^{a-1}(1+x)^{-a-b} e^{-c x} \mathbf{1}_{(0, \infty)}(x) d x, a, c>0, b \in \mathbb{R}
$$

( $C$ is the normalizing constant) and such that the law of $Y$ is the gamma distribution $\gamma(b, c)(d x)=\frac{c^{b}}{\Gamma(b)} x^{b-1} e^{-c x} \mathbf{1}_{(0, \infty)}(x) d x$, then the random variables

$$
U:=\frac{1+\frac{1}{X+Y}}{1+\frac{1}{X}}, \quad V:=X+Y
$$

are independent. Moreover, $U \sim \operatorname{Beta}(a, b)$ and $V \sim K^{(2)}(a+b,-b, c)$ where $\operatorname{Beta}(a, b)(d x)=$ $\frac{\Gamma(a+b)}{\Gamma(a) \Gamma(b)} x^{a-1}(1-x)^{b-1} \mathbf{1}_{\{0<x<1\}} d x$.

The Kummer and gamma distributions exist also as distributions on the set of positive definite matrices (the Wishart distribution in the gamma case), and matrix versions exist for the GIG-gamma Matsumoto-Yor property (see Letac and Wesołowski, 2000 and Massam and Wesołowski, 2006). Therefore, a natural question is whether the independence of the random variables $U$ and $V$ defined by (1.1) still holds if $X$ and $Y$ are $r \times r$ random matrices (of course, one has to formulate $U$ and $V$ as matrices, see (2.4)). We prove that the answer is affirmative. Although the proof only relies on a Jacobian calculation, the framework of matrices makes it non-trivial.

In Section 2 we state the result after a recall of the definition of matrix Kummer, Wishart and beta distributions. Section 3 is devoted to the proof and Section 4 to a short discussion.

\section{The result}

Let $r \geq 1$ be an integer. Denote by $\mathcal{M}_{r}$ the Euclidean space of $r \times r$ real symmetric matrices, and by $I$ the unit element of $\mathcal{M}_{r}$. Consider the inner product $\langle A, B\rangle=\operatorname{tr}(A B)$ on $\mathcal{M}_{r}$. Let $\mathcal{M}_{r}^{+}$be the cone of positive definite matrices in $\mathcal{M}_{r}$.

For $\Sigma \in \mathcal{M}_{r}^{+}$and $b \in\left\{0, \frac{1}{2}, 1, \frac{3}{2}, \ldots, \frac{r-1}{2}\right\} \cup\left(\frac{r-1}{2}, \infty\right)$, the Wishart distribution $\gamma(b, \Sigma)$ (see for instance Letac and Wesołowski, 2000 and Massam and Wesołowski, 2006) is defined as the law of a random matrix $Y$ valued in the closure of $\mathcal{M}_{r}^{+}$, with Laplace transform

$$
\mathbb{E}\left(e^{\langle\sigma, Y\rangle}\right)=\left(\frac{\operatorname{det} \Sigma}{\operatorname{det}(\Sigma-\sigma)}\right)^{b}, \quad \Sigma-\sigma \in \mathcal{M}_{r}^{+} .
$$

If $b>\frac{r-1}{2}$ (we refer to this case as the non-degenerate case), the Wishart distribution $\gamma(b, \Sigma)$ has a density:

$$
\gamma(b, \Sigma)(d y)=\frac{(\operatorname{det} \Sigma)^{b}}{\Gamma_{r}(b)}(\operatorname{det} y)^{b-(r+1) / 2} \exp (-\langle\Sigma, y\rangle) \mathbf{1}_{\mathcal{M}_{r}^{+}}(y) d y
$$


where $\Gamma_{r}$ is the multivariate gamma function defined for any complex number $z$ with $\operatorname{Re}(z)>(r-1) / 2$ by

$$
\Gamma_{r}(z)=\pi^{r(r-1) / 4} \prod_{j=1}^{r} \Gamma\left(z-\frac{j-1}{2}\right) .
$$

For $\alpha, \beta>\frac{r-1}{2}$, the beta distribution $\operatorname{Beta}(\alpha, \beta)$ (also called matrix variate beta of type I, see Nagar and Gupta, 2002) on $\mathcal{M}_{r}^{+}$is

$$
\operatorname{Beta}(\alpha, \beta)(d u)=\frac{\Gamma_{r}(\alpha+\beta)}{\Gamma_{r}(\alpha) \Gamma_{r}(\beta)}(\operatorname{det} u)^{\alpha-\frac{r+1}{2}}(\operatorname{det}(I-u))^{\beta-\frac{r+1}{2}} \mathbf{1}_{\mathcal{U}}(u) d u,
$$

where $\mathcal{U}$ is the set of matrices $u$ in $\mathcal{M}_{r}^{+}$such that $I-u \in \mathcal{M}_{r}^{+}$.

For $a>\frac{r-1}{2}, b \in \mathbb{R}, \Sigma \in \mathcal{M}_{r}^{+}$, the matrix Kummer distribution on $\mathcal{M}_{r}^{+}$is defined by

$$
K(a, b, \Sigma)(d x)=\frac{1}{\Gamma_{r}(a) \psi\left(a, a-b+\frac{r+1}{2} ; \Sigma\right)}(\operatorname{det} x)^{a-\frac{r+1}{2}}(\operatorname{det}(I+x))^{b} \exp (-\langle\Sigma, x\rangle) \mathbf{1}_{\mathcal{M}_{r}^{+}}(x) d x
$$

where $\psi$ is the confluent hypergeometric function of second kind with matrix argument (see Joshi and Joshi, 1985, formula (2)). This distribution is called in the literature (see e. g. Gupta et al, 2001) the Kummer-Gamma distribution or the Kummer distribution of type II. In this paper we concisely call it the Kummer distribution.

We now state the result of the present paper.

Theorem 2.1 Consider $a, b, \Sigma$ such that $a>\frac{r-1}{2}, b-a>\frac{r-1}{2}$ and $\Sigma \in \mathcal{M}_{r}^{+}$. Let $X$ and $Y$ be two independent random matrices valued in $\mathcal{M}_{r}^{+}$. Assume that $X$ follows the Kummer distribution $K(a, b, \Sigma)$ and $Y$ the Wishart distribution $\gamma(b-a, \Sigma)$.

Then, the random matrices

$$
U:=\left[I+(X+Y)^{-1}\right]^{1 / 2}\left[I+X^{-1}\right]^{-1}\left[I+(X+Y)^{-1}\right]^{1 / 2}, \quad V:=X+Y
$$

are independent. Furthermore, $U \sim \operatorname{Beta}(a, b-a)$ and $V \sim K(b, a, \Sigma)$.

\section{Proof}

For $x, y \in \mathcal{M}_{r}^{+}$define

$$
u:=\left[I+(x+y)^{-1}\right]^{1 / 2}\left[I+x^{-1}\right]^{-1}\left[I+(x+y)^{-1}\right]^{1 / 2}, \quad v:=x+y .
$$

Observe that, since $x$ and $y$ are positive definite matrices, it is also the case for $u, v$ and $I-u$. (It is obvious for $u$ and $v$. To check it for $I-u$, one can write

$$
I-u=\left[I+(x+y)^{-1}\right]^{1 / 2}\left(\left[I+(x+y)^{-1}\right]^{-1}-\left[I+x^{-1}\right]^{-1}\right)\left[I+(x+y)^{-1}\right]^{1 / 2}
$$

and use the fact that if $A$ and $B$ are positive definite matrices such that $A-B$ is positive definite, then $B^{-1}-A^{-1}$ is positive definite). Therefore, the transformation

$$
T:(x, y) \mapsto(u, v)
$$

maps $\mathcal{M}_{r}^{+} \times \mathcal{M}_{r}^{+}$to $\mathcal{U} \times \mathcal{M}_{r}^{+}$. It is clearly bijective. We now compute the Jacobian of $T^{-1}$. 


\subsection{The Jacobian}

It is convenient to write $T=T_{2} \circ T_{1}$, where $T_{1}: \mathcal{M}_{r}^{+} \times \mathcal{M}_{r}^{+} \rightarrow \mathcal{U} \times \mathcal{M}_{r}^{+}$and $T_{2}$ : $\mathcal{U} \times \mathcal{M}_{r}^{+} \rightarrow \mathcal{U} \times \mathcal{M}_{r}^{+}$are defined by

$$
T_{1}(x, y)=(w, z):=\left(\left[I+x^{-1}\right]^{-1}, x+y\right)
$$

and

$$
T_{2}(w, z)=(u, v):=\left(\left[I+z^{-1}\right]^{1 / 2} w\left[I+z^{-1}\right]^{1 / 2}, z\right) .
$$

We have

$$
(x, y)=T_{1}^{-1}(w, z)=\left(\left[w^{-1}-I\right]^{-1}, z-\left[w^{-1}-I\right]^{-1}\right)
$$

and

$$
(w, z)=T_{2}^{-1}(u, v)=\left(\left[I+v^{-1}\right]^{-1 / 2} u\left[I+v^{-1}\right]^{-1 / 2}, v\right) .
$$

Lemma 3.1 The Jacobian of the transformation $T^{-1}$ is

$$
J(u, v)=\operatorname{det}(I+v)^{\frac{r+1}{2}} \operatorname{det}(I+v-u v)^{-r-1} \operatorname{det} v^{\frac{r+1}{2}} .
$$

PROOF: We compute the Jacobian of $T^{-1}$ via the Jacobians $J_{(u, v) \rightarrow(w, z)}$ and $J_{(w, z) \rightarrow(x, y)}$.

$J_{(u, v) \rightarrow(w, z)}$ is the determinant of the differential of $T_{2}^{-1}$ at $(u, v)$. This differential is a linear endomorphism of $\mathcal{M}_{r} \times \mathcal{M}_{r}$ which, by (3.3), can be writen as

$$
\left(\begin{array}{cc}
D & E \\
0 & I d_{\mathcal{M}_{r}}
\end{array}\right)
$$

where $E$ does not need to be computed and $D$ is the differential of the function $u \mapsto[I+$ $\left.v^{-1}\right]^{-1 / 2} u\left[I+v^{-1}\right]^{-1 / 2}$ for fixed $v$. Thus, $J_{(u, v) \rightarrow(w, z)}$ equals $\operatorname{det} D$ (we use the same notation for the determinant of a matrix and the determinant of a linear operator). According to Muirhead (1982), Theorem 2.1.7, we have

$$
\operatorname{det} D=\left(\operatorname{det}\left[I+v^{-1}\right]^{-1 / 2}\right)^{r+1}=\left(\operatorname{det}\left[I+v^{-1}\right]\right)^{-\frac{r+1}{2}},
$$

i.e.

$$
J_{(u, v) \rightarrow(w, z)}=\left(\operatorname{det}\left[I+v^{-1}\right]\right)^{-\frac{r+1}{2}} .
$$

Let us now compute $J_{(w, z) \rightarrow(x, y)}$. By (3.2), we have $x=\left(\left[w^{-1}-I\right]^{-1}\right.$ so that the differential $G(w)$ of $x$ with respect to $w$ is the linear map

$$
h \mapsto\left(\left[w^{-1}-I\right]^{-1} w^{-1} h w^{-1}\left[w^{-1}-I\right]^{-1}\right.
$$

on $\mathcal{M}_{r}$. Its determinant is, again by Muirhead (1982), equal to

$$
\operatorname{det}\left(G(w)=\operatorname{det}\left(\left[w^{-1}-I\right]^{-1} w^{-1}\right)^{r+1}=\operatorname{det}(I-w)^{-(r+1)} .\right.
$$

Thus, by (3.2), we have

$$
J_{(w, z) \rightarrow(x, y)}=\operatorname{det}\left(\begin{array}{cc}
G(w) & 0 \\
H & I d_{\mathcal{M}_{r}}
\end{array}\right)=\operatorname{det}\left(G(w)=\operatorname{det}(I-w)^{-(r+1)}\right.
$$


where no detail is needed about $H$. It follows from (3.5)and (3.6) that

$$
J(u, v)=J_{(u, v) \rightarrow(w, z)} J_{(w, z) \rightarrow(x, y)}=\left(\operatorname{det}\left[I+v^{-1}\right]\right)^{-\frac{r+1}{2}} \operatorname{det}(I-w)^{-(r+1)} .
$$

We have

$$
\operatorname{det}\left[I+v^{-1}\right]=\operatorname{det}\left(v^{-1}\right) \operatorname{det}(I+v) .
$$

Since $\operatorname{det}(I+A B)=\operatorname{det}(I+B A)$ for any symmetric matrices $A$ and $B$, we write

$$
\begin{aligned}
\operatorname{det}(I-w) & =\operatorname{det}\left(I-\left[I+v^{-1}\right]^{-1 / 2} u\left[I+v^{-1}\right]^{-1 / 2}\right) \\
& =\operatorname{det}\left(I-u\left[I+v^{-1}\right]^{-1}\right) \\
& =\operatorname{det}\left(I-u v[I+v]^{-1}\right) \\
& =\operatorname{det}(I+v)^{-1} \operatorname{det}(I+v-u v)
\end{aligned}
$$

Substituting (3.8) and (3.9) in (3.7) we obtain (3.4).

\subsection{Density of $(U, V)$}

Denote by $f_{X}$ and $f_{Y}$ the densities of $X$ and $Y$ respectively, and by $f_{(U, V)}$ the density of $(U, V)$. Since $X$ and $Y$ are independent,

$$
f_{(U, V)}(u, v)=f_{X}(x) f_{Y}(y) J(u, v) \mathbf{1}_{\mathcal{U}}(u) \mathbf{1}_{\mathcal{M}_{r}^{+}}(v)
$$

where $(x, y)=T^{-1}(u, v)$ and $J(u, v)$ is the Jacobian given by (3.4). Recall that $X \sim$ $K(a, b, \Sigma)$ and $Y \sim \gamma(b-a, \Sigma)$. By (2.2) and (2.3), denoting by $\mathrm{C}$ the product of all constants, (3.10) can be written, for any $(u, v) \in \mathcal{U} \times \mathcal{M}_{r}^{+}$,

$$
\begin{aligned}
f_{(U, V)}(u, v)= & C(\operatorname{det} x)^{a-\frac{r+1}{2}}(\operatorname{det}(I+x))^{-b} \exp (-\langle\Sigma, x\rangle) \\
& \times(\operatorname{det} y)^{b-a-\frac{r+1}{2}} \exp (-\langle\Sigma, y\rangle) J(u, v) \\
= & C(\operatorname{det} x)^{a-\frac{r+1}{2}}(\operatorname{det}(I+x))^{-b}(\operatorname{det} y)^{b-a-\frac{r+1}{2}} \\
& \times \exp (-\langle\Sigma, x+y\rangle) J(u, v) .
\end{aligned}
$$

In the following lemma we express $\operatorname{det} x, \operatorname{det} y \operatorname{det}(I+x)$ in terms of $u$ and $v$.

Lemma 3.2 Consider $x, y, u, v$ such that $T(x, y)=(u, v)$ as in (3.1). Then,

$$
\begin{gathered}
\operatorname{det} x=\operatorname{det}\left((I+v-u v)^{-1}\right) \operatorname{det} u \operatorname{det} v, \\
\operatorname{det}(I+x)=\operatorname{det}\left((I+v-u v)^{-1}\right) \operatorname{det}(I+v), \\
\operatorname{det} y=\operatorname{det} v \operatorname{det}\left((I+v-u v)^{-1}\right) \operatorname{det}(I+v) \operatorname{det}(I-u) .
\end{gathered}
$$

PROOF: By (3.1) we have

$$
\begin{aligned}
x^{-1} & =\left(I+v^{-1}\right)^{1 / 2} u^{-1}\left(I+v^{-1}\right)^{1 / 2}-I \\
& =\left(I+v^{-1}\right)^{1 / 2}\left(u^{-1}-\left[I+v^{-1}\right]^{-1}\right)\left(I+v^{-1}\right)^{1 / 2},
\end{aligned}
$$


which gives

$$
x=\left(I+v^{-1}\right)^{-1 / 2}\left(u^{-1}-\left[I+v^{-1}\right]^{-1}\right)^{-1}\left(I+v^{-1}\right)^{-1 / 2} .
$$

Since $I+v^{-1}=v^{-1}(I+v)$ we have $u^{-1}-\left[I+v^{-1}\right]^{-1}=u^{-1}(I+v-u v)(I+v)^{-1}$, so that (3.15) gives

$$
\begin{aligned}
x & =v^{1 / 2}(I+v)^{-1 / 2}(I+v)(I+v-u v)^{-1} u v^{1 / 2}(I+v)^{-1 / 2} \\
& =v^{1 / 2}(I+v)^{1 / 2}(I+v-u v)^{-1} u v^{1 / 2}(I+v)^{-1 / 2}
\end{aligned}
$$

Taking the determinant we obtain (3.12).

The definitions of $u$ and $v$ imply

$$
\begin{aligned}
\operatorname{det} u & =\operatorname{det}\left(I+v^{-1}\right) \operatorname{det}\left(I+x^{-1}\right)^{-1} \\
& =(\operatorname{det} v)^{-1} \operatorname{det}(I+v) \operatorname{det} x \operatorname{det}(I+x)^{-1}
\end{aligned}
$$

from which we get $\operatorname{det}(I+x)=(\operatorname{det} u)^{-1}(\operatorname{det} v)^{-1} \operatorname{det}(I+v) \operatorname{det} x$. Then, (3.13) follows from (3.12).

To obtain (3.14), we use (3.16) to write

$$
\begin{aligned}
y & =v-x \\
& =v^{1 / 2} I v^{1 / 2}-v^{1 / 2}(I+v)^{1 / 2}(I+v-u v)^{-1} u(I+v)^{-1 / 2} v^{1 / 2} \\
& =v^{1 / 2}\left[I-(I+v)^{1 / 2}(I+v-u v)^{-1} u(I+v)^{-1 / 2}\right] v^{1 / 2} .
\end{aligned}
$$

As a consequence,

$$
\begin{aligned}
\operatorname{det} y & =\operatorname{det} v \operatorname{det}\left(I-(I+v-u v)^{-1} u\right) \\
& =\operatorname{det} v \operatorname{det}\left((I+v-u v)^{-1}(I+v-u v-u)\right)
\end{aligned}
$$

and (3.14) follows since $I+v-u v-u=(I-u)(I+v)$.

To complete the proof of the theorem, we plug (3.12), (3.13), (3.14) and (3.4) into (3.11), replace $x+y$ with $v$ and the result follows. Here are the details: for any $(u, v) \in$ $\mathcal{U} \times \mathcal{M}_{r}^{+}$

$$
\begin{aligned}
f_{(U, V)}(u, v)= & C(\operatorname{det}(I+v-u v))^{\frac{r+1}{2}-a}(\operatorname{det} u)^{a-\frac{r+1}{2}}(\operatorname{det} v)^{a-\frac{r+1}{2}} \\
& \times(\operatorname{det}(I+v-u v))^{b}(\operatorname{det}(I+v))^{-b} \\
& (\operatorname{det} v)^{b-a-\frac{r+1}{2}}(\operatorname{det}(I+v-u v))^{a-b+\frac{r+1}{2}}(\operatorname{det}(I+v))^{b-a-\frac{r+1}{2}}(\operatorname{det}(I-u))^{b-a-\frac{r+1}{2}} \\
& (\operatorname{det}(I+v))^{\frac{r+1}{2}}(\operatorname{det}(I+v-u v))^{-r-1}(\operatorname{det} v)^{\frac{r+1}{2}} \exp (-\langle\Sigma, v\rangle) \\
= & C(\operatorname{det} u)^{a-\frac{r+1}{2}}(\operatorname{det}(I-u))^{b-a-\frac{r+1}{2}}(\operatorname{det} v)^{b-\frac{r+1}{2}}(\operatorname{det}(I+v))^{-a}
\end{aligned}
$$

from which we see that $U$ and $V$ are independent and follow the laws $\operatorname{Beta}(a, b-a)$ and $K(b, a, \Sigma)$ respectively (see $(2.2)$ and $(2.3)$. 


\section{Discussion}

1. As said in the introduction, the independence property established in the present paper has been proved in the case $r=1$ by Koudou and Vallois (2010), where the converse has been proved too, thus providing a characterization of the product of Kummer and gamma laws under the assumption of existence and smoothness of densities. It is highly likely, although not easy to prove, that this characterization holds also in the case of matrices. Namely, one can make the following conjecture:

Let $X$ and $Y$ be two independent random matrices valued in $\mathcal{M}_{r}^{+}$with smooth densities. The random matrices $U$ and $V$ defined by (2.4) are independent if and only if there exist $a, b, \Sigma$ such that $a>\frac{r-1}{2}, b-a>\frac{r-1}{2}, \Sigma \in \mathcal{M}_{r}^{+}$and such that $X$ follows the Kummer distribution $K(a, b, \Sigma)$ and $Y$ the Wishart distribution $\gamma(b-a, \Sigma)$.

2. The Matsumoto-Yor property has been discovered in a particular case by Matsumoto and Yor (2001) while studying certain exponential functionals of the Brownian motion. An interesting question is whether there exists a stochastic process whose behaviour could be linked to the independence property shown in the present paper in the case $r=1$ as well as in the matrix framework.

\section{References}

[1] Gupta, A. K., Cardeno, L. and Nagar, D. K.(2001). Matrix-variate KummerDirichlet distributions. J. Applied. Math., 117-139.

[2] Joshi, R. M. and Joshi, J. M. C. (1985). Confluent hypergeometric function of second kind with matrix argument. Indian J. pure appl. Math. 16 (6), 627-636.

[3] Koudou, E. and Vallois, P. (2010). Independence properties of the Matsumoto-Yor type. To appear in Bernoulli.

[4] Koudou, E. and Vallois, P. (2011). Which distributions have the Matsumoto-Yor property? To appear in Electronic Communications in Probability.

[5] Letac, G. and Wesołowski, J. (2000). An independence property for the product of GIG and gamma laws. Ann. Prob. 28, 1371-1383.

[6] Massam, H. and Wesołowski, J. (2006). The Matsumoto-Yor property and the structure of the Wishart distribution. Journal of Multivariate Analysis 97, 103123.

[7] Matsumoto, H. and Yor, M. (2001). An analogue of Pitman's $2 M-X$ theorem for exponential Wiener functional, Part II: the role of the generalized inverse Gaussian laws. Nagoya Math. J. 162, 65-86.

[8] Muirhead, R. J. (1982). Aspects of Multivariate Statistical Theory. Wiley, New York. 
[9] Nagar and Gupta (2002). Matrix-variate Kummer-beta distributions. J. Austral. Math. Soc. 73, 11-25. 